\title{
Kinetically Favorable Growth of Oxides Nanowires and Pyramids with Gold Seeds
}

Guo-zhen Zhu ${ }^{1}$, Minghui Lin ${ }^{1}$, Yushun Liu ${ }^{1}$, Dong Yue Xie ${ }^{2}$ and Si-yu Yao ${ }^{2}$

${ }^{1}$ University of Manitoba, Winnipeg, Manitoba, Canada, ${ }^{2}$ Shanghai Jiao Tong University, Shanghai, Shanghai, China (People's Republic)

Oxide nanowires [1] have been identified as active components in solar cells, nanoelectromechanical devices, biosensors, chemical sensors, highly efficient catalysts, and lithium ion batteries because they have superior chemical, optical, electrical, and mechanical properties originating from their excellent crystalline quality. Vapor-phase synthesis, particularly vapor-liquid-solid (VLS) growth [2], is an undoubtedly promising and broadly adoptable approach to produce single-crystal silicon-based and other semiconducting nanowires in a controlled fashion. Vapor-phase synthesis can be potentially extended to the assembly of single-crystal oxide nanowires but with much less successful examples under complex circumstances. In order to produce single-crystal nanowires for a large range of oxides, a throughout investigation, based on much simple but effective growth settings, is demanded to understand and master the growth process for oxide nanowires.

We recently report a simple experimental setting of assembling single-crystal oxide nanowires, with uniform morphology over tens of microns in length [3-4]. More importantly, the prepared titanium oxide nanowires have surprisingly high materials quality, in term of compositional purity, structural perfection, geometrical uniformity (see Fig.1a). Oxide pyramids form prior to the growth of nanowires, suggesting a competinggrowth of nanowires and pyramids at the early stage. The formation of such pyramids can be different from those semiconducting pyramids observed in conventional VLS growth which originate from the changing solubility of vapor species in liquid seeds [5].

As shown in the experimental setting illustrated in Fig.1b, our approach only involves a heat treatment of gold seeds-deposited oxide substrates, which are sealed in a quartz tube. Because the single-crystal substrate serves as the source for vapor species through thermal vaporization, we believe that the growth of nanowires is kinetically favorable, and the enrichment of vapor species likely occurs at the seed surfaces followed by the transfer to the growth front. In order to verify this mechanism, titanium oxide are used as the model system, which has the dominant vapor species at elevated temperatures being oxide vapors. A thin layer of $\sim 2 \mathrm{~nm}$ thickness, is observed at the seed surface, with a notable amount of titanium and oxygen EDX signals. This thin layer is further investigated by an in-situ TEM experiment in which nanowires are heated to the growth temperature, as shown in the heating profile in Fig.1c [3]. The thin layer has no visible change over time at $600{ }^{\circ} \mathrm{C}$. While held at $800{ }^{\circ} \mathrm{C}$, the thin layer disappears within a few minutes and there are significant material increments and shape changes at the growth front. The current results suggest a possible transfer of the thin layer materials to the growth front.

In order to deeply study the growth process and to enhance the adaptability and controllability of this simple synthesis method, further in-situ experiments are required to assess the morphology changes at the growth front, for the nanowires and the pyramids [6]. 

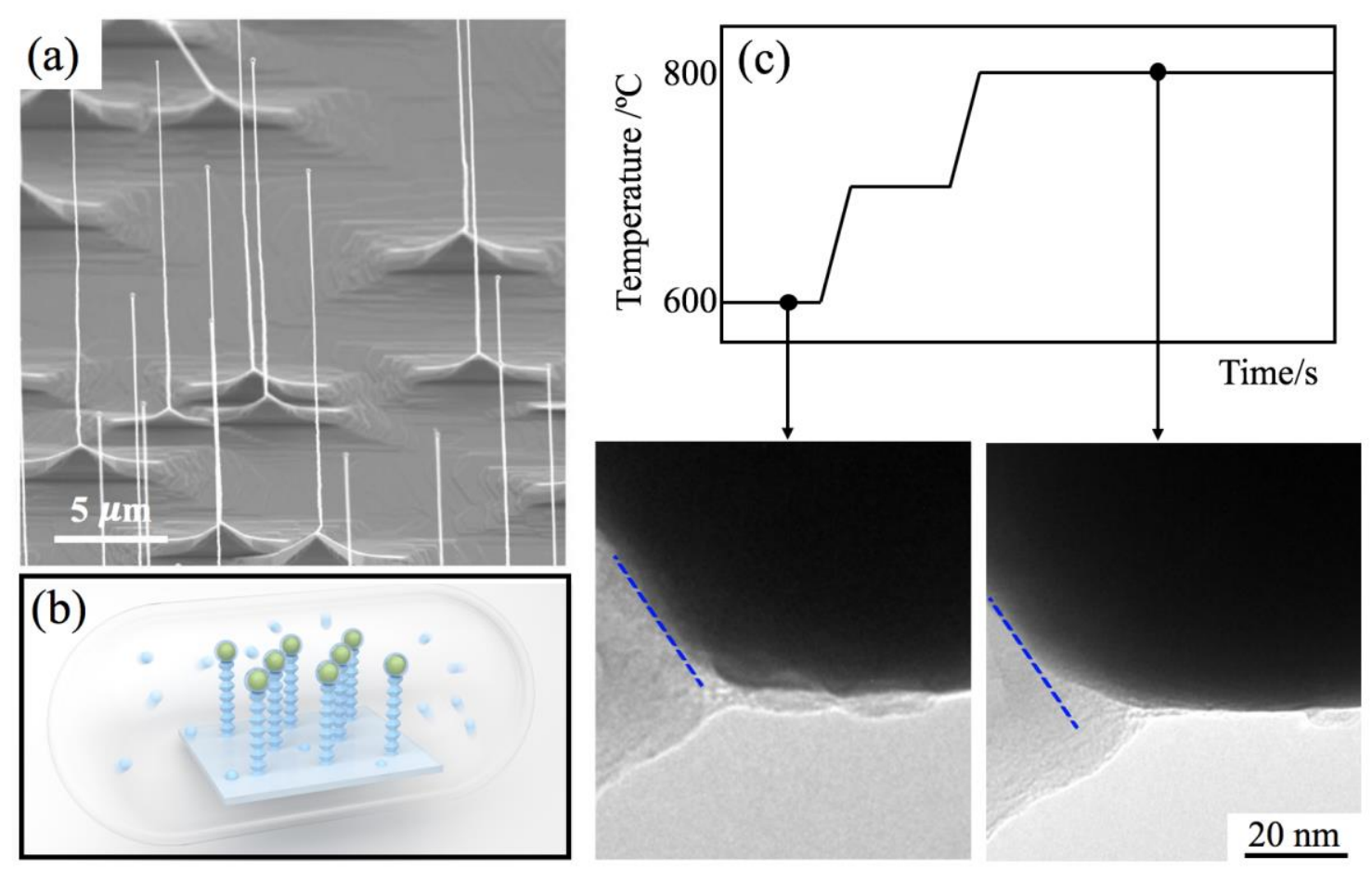

Figure 1. (a) Typical morphology of TiO2nanowires; (b) Experimental setting including Au-coated TiO2substrates heated at elevated temperatures; (c) In-situ heating experiment tracking the thin layer at the seed surface.

\section{References}

[1] A. Zhang, G. Zheng, and C. Lieber, Nanowires: Building Blocks for Nanoscience and Nanotechnology (2016).

[2] R.S. Wagner and W.C. Ellis, Appl. Phys. Lett. 4 (1964), pp.89-90.

[3] D.Y.Xie, et al., Small Methods 3(2019),p.1900111.

[4] M. Lin, et al.,ACS Omega5 (2020), pp. 1566-1571.

[5] A. Yi, et al., Mater. Charact. 151 (2019), pp.237-241.

[6] The authors acknowledge the support from Canada Research Chair Program and University of Manitoba. 